\title{
Representations Obeying the Spectrum Condition
}

\author{
Sergio Doplicher and Mauro Spera \\ Istituto Matematico "G. Castelnuovo", Università di Roma, Rome, Italy
}

\begin{abstract}
We show that every properly infinite, injective von Neumann algebra acting on a separable Hilbert space is isomorphic to the weak closure of some translation covariant representation, obeying the spectrum condition for the generators of the translation group, of the $C^{*}$-algebra of quasilocal observables of a free massless spinor field. We construct explicitly such representations in the case of $I I_{\infty}$ and $I I I_{\lambda}$ factors, $0<\lambda<1$.
\end{abstract}

\section{Introduction}

The von Neumann algebra generated by a representation $\pi$ of the $C^{*}$ algebra $\mathfrak{A}$ of quasilocal observables of a local quantum theory [1] is known to be type $I$ if $\pi$ is covariant for the space time translation group, the representation $\mathscr{U}$ of this group on $\mathscr{H}_{\pi}$ fulfills the spectrum condition

$$
\operatorname{Sp}(\mathscr{U}) \subset \bar{V}_{+}
$$

and there is a $\mathscr{U}$-invariant vector which is cyclic for $\pi$ (the vacuum) [2]. In absence of the vacuum, $\pi(\mathfrak{U})^{\prime \prime}$ is also type $I$ if the spectrum condition (1.1) is sharpened by requiring the existence of a massive particle isolated from the rest of the spectrum [3].

We show that in presence of massless particles all types of von Neumann algebras can appear among the positive energy representations of $\mathfrak{X}$. This answers a question posed by D. Buchholz.

We study a simple model, the even part of the field algebra of a free massless Majorana particle. Specifically, we consider the CAR algebra $\mathfrak{A}(K)$ over $K$, when $K$ is the direct sum of the Hilbert spaces of the irreducible unitary representations of the covering of the Poincaré group of zero mass, spin $1 / 2$ and helicities \pm . The destruction and creation operators $a(f), a(g)^{*}, f, g \in K$, fulfilling the CAR, are related in the standard way to the negative and positive frequency parts of the free massless Majorana field $\psi{ }^{1}$ The local field algebras $\mathfrak{F}(\mathcal{O})$ are the $C^{*}$-subalgebras of $\mathfrak{U}(K)$

1 By considering $K \oplus K$ instead of $K$ to allow distinction between particles and antiparticles, we could similarly study a massless Dirac theory 
generated by $\psi$ regularized with test functions with support in a given region $\mathcal{O}$. The gauge transformation to the angle $\pi$ defines an automorphism $\gamma$ of $\mathfrak{A}(K)$ such that

$$
\gamma(a(f))=-a(f) ; \quad \gamma(\psi)=-\psi
$$

The $\gamma$-fixed point subalgebras $\mathfrak{B}(\mathcal{O})$ of $\mathfrak{F}(\mathcal{O})$ provide a model of local quantum theory fulfilling the axioms in [1], where the quasilocal observable algebra $\mathfrak{B}=$ $\mathfrak{U}(K)_{e}$ is simple, separable, the action of the Poincare group is strongly continuous [4], [5]; moreover $\mathfrak{B}$ is isomorphic to the CAR algebra itself [6].

We will neglect specifying the spin indices since they will play no role here. Our method is a generalization to the non type $I$ case of the construction given in [7] of positive energy irreducible representations of $\mathfrak{U}(K)$ without a vacuum.

This construction consists in choosing a special orthonormal set $g_{1}$, $g_{2} \ldots g_{n} \ldots \in K$ such that

$$
\begin{aligned}
& \text { supp } \tilde{g}_{n} \subset\left\{\mathbf{k} /|\mathbf{k}| \leqq \varepsilon_{n}\right\}, \quad n=1,2, \ldots ; \\
& \text { supp } \tilde{g}_{n} \cap \operatorname{supp} \tilde{g}_{m}=\varnothing \quad \text { if } \quad n \neq m, \\
& \sum_{n=1}^{\infty} \varepsilon_{n}<\infty .
\end{aligned}
$$

Then ([7]) every discrete representation of the CAR algebra over $K$ generated by a state with infinitely many of the modes $g_{1}, g_{2} \ldots$ occupied and all the remaining modes empty, is an irreducible positive energy representation. This method of construction has been generalized in various ways (see e.g. $[8,9]$ ).

Let $N_{1}$ denote the closed subspace of $K$ generated by $g_{1}, g_{2} \ldots$ and $N_{2}=N_{1}^{\perp}$. The key point is that any representation of $\mathfrak{Q}(K)$, which restricts to a multiple of the Fock representation on $\mathfrak{U}\left(N_{2}\right)$, is covariant with positive energy. This is to be expected intuitively since, if we choose a reference state whose restriction to $\mathfrak{U}\left(N_{2}\right)$ is the Fock vacuum, the energy of this state should be bounded by $\sum \varepsilon_{n}<\infty$.

Note that with evident modifications on conditions (1.3), specifying also the spin variables, we could arrange that all our representations are rotation covariant. However covariance under boosts is clearly impossible but for the Fock representation.

In Sect. 2 we prove by abstract arguments that every injective properly infinite von Neumann algebra on a separable Hilbert space appears as the weak closure of a positive energy representation of $\mathfrak{B}$, which is Fock-like on $\mathfrak{U}\left(N_{2}\right)_{e}$. In Sect. 3 we discuss some explicit examples; we choose states where each mode $g_{i}$ is occupied with a constant probability $\mu$ and each mode in $N_{2}$ is empty. These states are a simple variant of Powers states and for $0<\mu<1 / 2$, respectively $\mu=1 / 2$, they generate factor representations of $\mathfrak{B}$ of all types $I I I_{\lambda}, 0<\lambda<1$, respectively of type $I I_{\infty}$, which are covariant with positive energy. Note that each covariant representation of $\mathfrak{U}(K)$ with positive energy is locally normal $[10,11]$.

It is natural to ask whether results similar to ours apply to a general interacting theory, provided there are massless particles and each vector state in the vacuum sector below some energy threshold can be approximated by asymptotic states of finitely many massless particles [12]. We do not discuss this question here. It can 
however be expected that representations like those constructed here are not generated by scattering states unless one considers very artificial incoming states for the massless particles.

\section{Abundance of Positive Energy Representations}

We consider only CAR algebras over separable Hilbert spaces. We denote as above by $\mathfrak{U}(M)$ the CAR algebra over a Hilbert space $M$, by $\mathfrak{A}(M)_{e}=\{A \in \mathfrak{U}(M) / \gamma(A)=A\}$ its even part; we will say that a representation $\pi$ of $\mathfrak{U}(M)$ is even if it is quasiinvariant under $\gamma$, i.e. $\pi \circ \gamma \approx \pi$. In this case $\tilde{\gamma}$ will denote the automorphism of $\pi(\mathfrak{H})^{\prime \prime}$ such that $\tilde{\gamma}(\pi(A))=\pi \circ \gamma(A), A \in \mathfrak{A}(M)$. The Fock representation will be denoted by $\pi_{F}$, acting on $\mathscr{H}_{F}$.

If $M=N_{1} \oplus N_{2}$ and $\gamma_{1}, \gamma_{2}$ denote the automorphism of $\mathfrak{U}(M)$ generated by the unitaries $(-I) \oplus I, I \oplus(-I)$ respectively, on $N_{1} \oplus N_{2}$, the subalgebra of $\gamma_{1}, \gamma_{2}$-fixed points in $\mathfrak{U}(M)$ is generated by $\mathfrak{U}\left(N_{1}\right)_{e} \cup \mathfrak{U}\left(N_{2}\right)_{e}$, and is isomorphic to the (unique) $C^{*}$ tensor product $\mathfrak{A}\left(N_{1}\right)_{e} \otimes \mathfrak{A}\left(N_{2}\right)_{e}$. The map $\eta=(1 / 4)\left(1+\gamma_{1}+\gamma_{2}+\gamma\right)$ provides a conditional expectation of $\mathfrak{U}(M)$ onto the $\gamma_{1}, \gamma_{2}$-fixed points.

2.1. Lemma. Let $\pi$ be an even representation of $\mathfrak{U}(K), K=N_{1} \oplus N_{2}$, with $N_{2}$ infinite dimensional. If

$$
\pi \mid \mathfrak{U}\left(N_{2}\right) \approx \pi_{F}
$$

then

$$
\pi\left(\mathfrak{A}(K)_{e}\right)^{\prime \prime} \cong \pi\left(\mathfrak{U}\left(N_{1}\right)_{e}\right)^{\prime \prime} \bar{\otimes} \mathfrak{B}\left(\mathscr{H}_{F}^{e}\right) .
$$

Proof. Since $\pi$ is even and by (2.1), $\gamma, \gamma_{2}$ and $\gamma_{1}=\gamma \gamma_{2}$ are normal in the representation $\pi$. Let $\mathscr{R}, \mathscr{R}_{i}, \mathscr{R}_{e}, \mathscr{R}_{i e}$ denote the weak closures of $\pi(\mathfrak{U}(K)), \pi\left(\mathfrak{U}\left(N_{i}\right)\right)$ and of their even parts respectively. The normal extensions of $\gamma_{i}, \eta$ to $\mathscr{R}_{i}$ and $\mathscr{R}$ respectively will be denoted by $\tilde{\gamma}_{i}, \tilde{\eta}$, so that $\mathscr{R}_{i e}=\mathscr{R}_{i}^{\hat{\gamma}_{t}}$ and $\tilde{\eta}(\mathscr{R})=\mathscr{R}_{1 e} \vee \mathscr{R}_{2 e}$.

By (2.1) there is a self-adjoint unitary $U_{0} \in \mathscr{R}_{2}$ such that

$$
U_{0} A U_{0}^{-1}=\tilde{\gamma}_{2}(A), A \in \mathscr{R}_{2} .
$$

Let $U_{0}=E_{0}-F_{0}$ be the spectral resolution of $U_{0}$; by (2.1) $\mathscr{R}_{2}$ is a type $I$ factor, and since $\mathscr{R}_{1 e} \subset \mathscr{R}_{2}^{\prime}$, we have

$$
\begin{aligned}
E_{0} \mathscr{R}_{e} E_{0} & =E_{0} \tilde{\eta}(\mathscr{R}) E_{0}=\mathscr{R}_{1 e} E_{0} \vee E_{0} \mathscr{R}_{2} E_{0} \\
& \cong \mathscr{R}_{1 e} E_{0} \otimes \mathfrak{B}\left(\mathscr{H}_{F}^{e}\left(N_{2}\right)\right),
\end{aligned}
$$

denoting by $\mathscr{H}_{F}^{e}\left(N_{2}\right)$ the even subspace of the Fock space over $N_{2}$. Since $N_{2}$ is infinite dimensional, $E_{0}, F_{0}$ are infinite projections in the type $I_{\infty}$ factor $\mathscr{R}_{2}$; hence there is $W_{0} \in \mathscr{R}_{2}$ such that

$$
W_{0} W_{0}^{*}=E_{0}, W_{0}^{*} W_{0}=I
$$

Since $\mathscr{R}_{1 e} \subset \mathscr{R}_{2}^{\prime}$ by (2.5) we have that $\mathscr{R}_{1 e} E_{0}$ and $\mathscr{R}_{1 e}$ are unitarily equivalent. By (2.4), the Lemma will be proved if we show that $E_{0} \mathscr{R}_{e} E_{0}$ and $\mathscr{R}_{e}$ are unitarily equivalent. This is the case if $E_{0} \sim I \bmod \mathscr{R}_{e}$, i.e. there is an isometry $W \in \mathscr{R}_{e}$ fulfilling 
(2.5). We construct such a $W$ by twisting $W_{0}$ as follows. Note that

$$
\tilde{\gamma}\left(W_{0}\right)=U_{0} W_{0} U_{0}^{-1}=W_{0}\left(E_{0}-F_{0}\right) .
$$

Let $f \in N_{1},\|f\|=1, U_{f}=a(f)+a(f)^{*} ; \pi\left(U_{f}\right)$ commutes with $\mathscr{R}_{2 e}$ hence with $E_{0}, F_{0}$, the operator $E_{0}+F_{0} \pi\left(U_{f}\right)$ is self-adjoint and unitary, and by multiplying $W$ with a unitary on the right we still fulfill (2.5); by setting

we have

$$
W=W_{0}\left(E_{0}+F_{0} \pi\left(U_{f}\right)\right) \in \mathscr{R}
$$

$$
\begin{aligned}
\tilde{\gamma}(W) & =\tilde{\gamma}\left(W_{0}\right)\left(E_{0}+F_{0} \tilde{\gamma}\left(\pi\left(U_{f}\right)\right)\right) \\
& =W_{0}\left(E_{0}-F_{0}\right)\left(E_{0}-F_{0} \pi\left(U_{f}\right)\right)=W ;
\end{aligned}
$$

hence $W$ fulfills $(2.5)$ and belongs to $\mathscr{R}_{e}$.

2.2. Lemma. Let $\mathscr{R}$ be any properly infinite injective von Neumann algebra on a separable Hilbert space, and $K=N_{1} \oplus N_{2}$ with $N_{1}, N_{2}$ infinite dimensional. There is a state $\omega$ on $\mathfrak{U}(K)_{e}$ such that

$$
\begin{aligned}
& \pi_{\omega}\left(\mathfrak{U}(K)_{e}\right)^{\prime \prime} \text { is isomorphic to } \mathscr{R}, \\
& \omega \mid \mathfrak{U}\left(N_{2}\right)_{e}=\omega_{F} .
\end{aligned}
$$

Proof. By a theorem of O. Marechal [13] there is a representation $\pi$ of $\mathscr{U}\left(N_{1}\right)_{e}$ such that $\pi\left(\mathfrak{U}\left(N_{1}\right)_{e}\right)^{\prime \prime}=\mathscr{R}$; then $\pi$ has a cyclic vector and there is a state $\varphi$ over $\mathfrak{U}\left(N_{1}\right)_{e}$ such that $\pi \cong \pi_{\varphi}$. With $\omega_{F}$ the Fock state on $\mathfrak{U}\left(N_{2}\right)_{e}$ define

$$
\tilde{\omega}=\left(\varphi \otimes \omega_{F}\right) \circ \eta
$$

then the restriction of $\tilde{\omega}$ to $\mathfrak{A}\left(N_{2}\right)$ is the Fock state and $\pi_{\tilde{\omega}} \mid \mathfrak{A}\left(N_{2}\right) \approx \pi_{F}$.

By Lemma 2.1

$$
\pi_{\tilde{\omega}}\left(\mathfrak{A}(K)_{e}\right)^{\prime \prime} \cong \pi_{\tilde{\omega}}\left(\mathfrak{A}\left(N_{1}\right)_{e}\right)^{\prime \prime} \bar{\otimes} \mathfrak{B}(\mathscr{H}),
$$

with $\mathscr{H}$ a separable Hilbert space.

Since $\tilde{\omega}$ extends $\varphi$ by (2.7), $\pi_{\varphi}$ is a subrepresentation of $\pi_{\tilde{\omega}} \mid \mathfrak{A}\left(N_{1}\right)_{e}$ and by (2.8) there is a projection $P \in \pi_{\tilde{\omega}}\left(\mathfrak{U}(K)_{e}\right)^{\prime}$ such that the induced subalgebra $\pi_{\tilde{\omega}}\left(\mathfrak{U}(K)_{e}\right)^{\prime \prime} P$ is isomorphic to $\mathscr{R} \otimes \mathfrak{B}(\mathscr{H})$, and hence to $\mathscr{R}$, since $\mathscr{R}$ is properly infinite. With $\widetilde{\xi}$ the GNS vector of $\tilde{\omega}$ in the representation $\pi_{\tilde{\omega}}$, the desired state $\omega$ is induced on $\pi_{\tilde{\omega}} \mid \mathfrak{H}(K)_{e}$ by the unit vector $(\tilde{\xi}, P \tilde{\xi})^{-1 / 2} P \tilde{\xi}$. For, condition (i) is fulfilled by construction and $\omega \mid \mathfrak{A}\left(N_{2}\right)_{e}$ is dominated by $\omega_{F}$, hence coincides with it by purity of $\omega_{F}$.

2.3. Lemma. Let $K$ be, as in the Introduction, the one particle space for the free Majorana field, and $K=N_{1} \oplus N_{2}$ with $N_{1}$ generated by an infinite orthonormal set fulfilling the conditions (1.3). For any even state $\omega$ on $\mathfrak{U}(K)$ such that $\omega \mid \mathfrak{A}\left(N_{2}\right)$ is the Fock state, $\pi_{\omega}$ is a covariant representation fulfilling the spectrum condition (1.1).

Proof. Since $\omega=\omega \circ \gamma$ and $\omega \mid \mathfrak{U}\left(N_{2}\right)_{e}=\omega_{F}$, with $\varphi=\omega \mid \mathfrak{U}\left(N_{1}\right)_{e}$, it is easily seen that

$$
\omega=\left(\varphi \otimes \omega_{F}\right) \circ \eta \text {. }
$$

By a theorem of Glimm ([14]; see also $[15,11 \cdot 2 \cdot 1])$ there is a sequence of finite particle unit vectors $x_{i}$ from $\mathscr{H}_{F}^{e}\left(N_{1}\right)$ such that $\varphi_{i} \equiv \omega_{x_{i}} \circ \pi_{F}$ converge to $\varphi$ in the 
weak-* topology of $\mathfrak{U}\left(N_{1}\right)_{e}^{*}$ as $i \rightarrow \infty$. Then the sequence of states on $\mathfrak{U}(K)$ given by

$$
\omega_{i}=\left(\varphi_{i} \otimes \omega_{F}\right) \circ \eta
$$

converges to $\omega$, given by (2.9) in the weak-* topology of $\mathfrak{A}(K)^{*}$ as $i \rightarrow \infty$.

Let $\tilde{x}_{i}$ denote the image of $x_{i}$ under the canonical immersion of $\mathscr{H}_{F}^{e}\left(N_{1}\right)$ into $\mathscr{H}_{F}(K)$; then $\omega_{i}$ is the vector state of $\mathfrak{U}(K)$ induced by $\tilde{x}_{i}$ in the Fock representation. The vectors $\tilde{x}_{i}$ are obtained from the vacuum creating finitely many particles in the modes $g_{1}, g_{2}, \ldots$; by the exclusion principle and by conditions (1.3) each $\tilde{x}_{i}$ has energy spectrum in $[0, \varepsilon], \varepsilon=\sum_{n=1}^{\infty} \varepsilon_{n}$, hence belongs to the spectral subspace for the energy momentum operators relative to the compact set $\left\{k \in \mathbb{R}^{4} / 0 \leqq k_{0} \leqq \varepsilon,|\mathbf{k}| \leqq \varepsilon\right\}$.

By a result of Borchers [16], the weak* limit $\omega$ of $\omega_{x_{1}}{ }^{\circ} \pi_{F}=\omega_{i}$ generates a covariant representation obeying the spectrum condition (1.1).

2.4. Theorem. Let $\mathscr{R}$ be a properly infinite injective von Newmann algebra on a separable Hilbert space; there is a representation $\pi$ of the $C^{*}$ algebra $\mathfrak{B}$ of quasilocal observables for the free massless Majorana field, which fulfills

(i) $\pi$ is a locally normal, translation covariant representation obeying the spectrum condition (1.1);

(ii) $\pi(\mathfrak{B})^{\prime \prime}$ is isomorphic to $\mathscr{R}$.

Proof. With $\omega$ the state of $\mathfrak{B}=\mathfrak{A}(K)_{e}$ provided by Lemma 2.2. the even extension $\tilde{\omega}=\omega^{\circ}(1 / 2)(1+\gamma)$ of $\omega$ to $\mathfrak{U}(K)$ fulfills the conditions of Lemma 2.3 ; hence $\pi_{\tilde{\omega}}$ is covariant and fulfills the spectrum condition (1.1) (i.e. is a "positive" representation). Since $\tilde{\omega}$ extends $\omega, \pi=\pi_{\omega}$ is a subrepresentation of the restriction $\pi_{\tilde{\omega}} \mid \mathfrak{B}$. Since $\mathfrak{B}$ is globally stable under translations and subrepresentations of positive representations are positive by [17], $\pi$ is also a positive representation. Then local normality follows from [10], [11].

\section{Explicit examples}

We will need the following variant of a result in [4]. Notations are those of the beginning of Sect. 2 and Lemma 2.1.

3.1. Lemma. Let $\pi$ be an even factorial representation of $\mathfrak{U}(K)$ with weak closure $\mathscr{R}$. We have the following alternative: either

(i) $\mathscr{R}_{e}$ is a factor and $\tilde{\gamma}$ is outer;

or

(ii) $\mathscr{R}_{e}^{\prime} \cap \mathscr{R}_{e}=\mathbb{C I}+\mathbb{C} U$, with $U$ a unitary implementing $\tilde{\gamma}$ on $\mathscr{R}$. In this case $\mathscr{R}$ is infinite and

$$
\mathscr{R}_{e} \cong \mathscr{R} \oplus \mathscr{R}
$$

Proof. If $U \in \mathscr{R}$ is a unitary implementing $\tilde{\gamma}, \tilde{\gamma}(U)=U$ and $U \in \mathscr{R}_{e} \cap \mathscr{R}_{e}^{\prime}$. So if $\mathscr{R}_{e}$ is a factor, $\tilde{\gamma}$ is outer.

Let $B \in \mathscr{R}_{e}^{c} \equiv \mathscr{R}_{e}^{\prime} \cap \mathscr{R}$ and $f, g \in K,\|f\|=\|g\|=1$. We have $\pi\left(U_{f} U_{g}\right) \in \mathscr{R}_{e}$ and $\pi\left(U_{f} U_{g}\right) B=B \pi\left(U_{f} U_{g}\right)$, hence

$$
\pi\left(U_{f}\right) B \pi\left(U_{f}\right)=\pi\left(U_{g}\right) B \pi\left(U_{g}\right) \equiv \gamma_{0}(B) .
$$


Since $\gamma_{0}(B)$ does not depend upon $f$ by (3.2), we see that $\gamma_{0}(B) \in \mathscr{R}_{e}^{\prime}$ and $\gamma_{0}$ is a period 2 automorphism of $\mathscr{R}_{e}^{\prime} \cap \mathscr{R}$. The $\gamma_{0}$-fixed points are in $\mathscr{R}^{\prime} \cap \mathscr{R}$ by (3.2) hence are multiples of $I$. If $B_{0} \in \mathscr{R}_{e}^{\prime} \cap \mathscr{R}$ is not a multiple of $I, B=B_{0}-\gamma_{0}\left(B_{0}\right) \neq 0$ and

$$
\gamma_{0}(B)=-B
$$

then $B^{*} B, B B^{*}$ are $\gamma_{0}$ invariant hence multiples of $I$, and $B=z U, z \in \mathbb{C}$, with $U$ a unitary inducing $\tilde{\gamma}$ on $\mathscr{R}$ by (3.2), (3.3); we can choose the coefficient $z$ so that $U^{2}=I$.

With $A \in \mathscr{R}_{e}^{\prime} \cap \mathscr{R}$ we have

$$
A=\frac{1}{2}\left(A+\gamma_{0}(A)\right)+\frac{1}{2}\left(A-\gamma_{0}(A)\right)=\lambda I+\mu U .
$$

Hence either we have (i) and $\mathscr{R}_{e}^{c}=\mathbb{C} I$, or $\mathscr{R}_{e}^{c}=\mathscr{R}_{e}^{\prime} \cap \mathscr{R}_{e}=\mathbb{C} I+\mathbb{C} U$.

If $\mathscr{R}$ is finite we are in case (i). For $\pi$ is quasiequivalent to $\pi_{\varphi_{0}}$, with $\varphi_{0}$ the trace of $\mathfrak{A}(K)$. With $\sigma=\operatorname{Ad} V, V$ any odd unitary in $\mathfrak{A}(K)$, we have

$$
\pi_{\varphi_{0}} \mid \mathfrak{X}(K)_{e} \cong \pi_{\varphi_{0} \mid \mathfrak{I}(K)_{e}} \oplus \pi_{\varphi_{0} \circ \sigma \mid \mathfrak{I}(K)_{e}} .
$$

With $\rho$ a * isomorphism of $\mathfrak{U}(K)$ onto $\mathfrak{U}(K)_{e},([6])$, we have by (3.4)

$$
\pi_{\varphi_{0}} \circ \rho \cong \pi_{\varphi_{0} \circ \rho} \oplus \pi_{\varphi_{0} \circ \sigma \circ \rho} .
$$

By the uniqueness of the trace, $\varphi_{0} \circ \rho=\varphi_{0} \circ \sigma \circ \rho=\varphi_{0}$, hence $\pi_{\varphi_{0}} \circ \approx \pi_{\varphi_{0}}$ and

$$
\pi_{\varphi_{0}}\left(\mathfrak{U}(K)_{e}\right)^{\prime \prime}=\pi_{\varphi_{0}} \circ \rho(\mathfrak{U}(K))^{\prime \prime} \sim \pi_{\varphi_{0}}(\mathfrak{U}(K))^{\prime \prime},
$$

which is the hyperfinite $I I_{1}$ factor, isomorphic to $\mathscr{R}$. That is if $\mathscr{R}$ is finite, $\mathscr{R}_{e}$ is a factor.

If we are in case (ii), $I$ is an infinite projection; with $U=E-F$ the spectral resolution of $U$, by (3.3) we have

$$
\pi\left(U_{f}\right) E \pi\left(U_{f}\right)=F
$$

hence $E$ and $F$ are equivalent $\bmod \mathscr{R}$; since $E+F=I, E$ and $F$ are infinite and $\mathscr{R}_{E} \cong$ $\mathscr{R}, \mathscr{R}_{F} \cong \mathscr{R}$ then (3.1) follows from $\mathscr{R}_{e}=\mathscr{R}_{E}+\mathscr{R}_{F}$.

With $A$ a positive contraction on $K$, let $\omega_{A}$ denote the gauge invariant quasifree state of $\mathfrak{A}(K)$ defined by $A$ setting (see e.g. [18]):

$$
\begin{aligned}
& \omega_{A}\left(a\left(f_{n}\right)^{*} \ldots a\left(f_{1}\right)^{*} a\left(g_{1}\right) \ldots a\left(g_{m}\right)\right) \\
& \quad=\delta_{m m} \operatorname{det}\left\{\left(f_{i}, A g_{k}\right)\right\}_{\substack{i=1,2 \ldots n \\
k=1,2 \ldots m}} ; f_{i}, g_{k} \in K .
\end{aligned}
$$

Taking $A=\lambda I, 0<\lambda \leqq 1 / 2$ one gets the Powers states; the von Neumann algebra $\mathscr{R}^{(\lambda)}$ generated by $\omega_{\lambda I}$ is the injective factor of type $I I I_{\lambda /(1-\lambda)}$ for $0<\lambda<1 / 2$ and the hyperfinite $I I_{1}$ factor for $\lambda=1 / 2$. (The injective factors of type $I I_{\infty}$ and $I I I_{\lambda}$, $0 \leqq \lambda \leqq 1$ are unique by the work of Alain Connes; see [19].)

The modular automorphisms $\sigma_{t}$ of $\mathscr{R}^{(\lambda)}$ determined by the GNS vector $\xi_{\lambda}$ of $\omega_{\lambda I}$ are induced by the one parameter unitary group

$$
f \in K \rightarrow\left(\frac{1-\lambda}{\lambda}\right)^{\text {it }} f, \quad t \in \mathbb{R},
$$

as is easily checked with the help of (3.5) by verifying the KMS condition [22], [23], [24]. 
In particular

$$
\tilde{\gamma}=\sigma_{t_{0}} \text { if }\left(\frac{1-\lambda}{\lambda}\right)^{\mathrm{it}_{0}}=-1 \text {. }
$$

3.2. Proposition. The von Neumann algebra $\mathscr{R}_{e}^{(\lambda)}$ generated by the even subalgebra in the Powers representation is the injective factor of type $I I I_{(\lambda /(1-\lambda))^{2}}$ for $0<\lambda<1 / 2$ and the hyperfinite $I I_{1}$ factor for $\lambda=1 / 2$.

Proof. The case $\lambda=1 / 2$ follows from the proof of Lemma 2.1. Let $0<\lambda<1 / 2$.

By Eq. (3.7) $\mathscr{R}_{e}^{(\lambda)}$ is stable under $\sigma_{t}$, hence the modular automorphisms of $\mathscr{R}_{e}^{(\lambda)}$ determined by the faithful normal state induced by $\xi_{\lambda}$ are the restrictions of $\sigma_{t}, t \in \mathbb{R}$, to $\mathscr{R}_{e}^{(\lambda)}[20],[23]$. Hence the modular operator $\Delta_{e}$ of $\left(\mathscr{R}_{e}^{(\lambda)}, \xi_{\lambda}\right)$ is the restriction of the modular operator $\Delta$ of $\left(\mathscr{R}^{(\lambda)}, \xi_{\lambda}\right)$ to the even subspace of $\pi_{\omega_{, I}}$ and by (3.6)

$$
\begin{aligned}
\operatorname{Sp}(\Delta) & =\left(\frac{1-\lambda}{\lambda}\right)^{\mathbb{Z}} \cup\{0\} \\
\operatorname{Sp}\left(\Delta_{e}\right) & =\left(\frac{1-\lambda}{\lambda}\right)^{2 \mathbb{Z}} \cup\{0\} .
\end{aligned}
$$

Since $\omega_{\lambda I}$ is invariant under permutation of factors in the tensor product description of $\mathfrak{U}(K)$ and $\gamma$ commutes with such permutations, by [21, th.1] we have

$$
\begin{aligned}
& S\left(\mathscr{R}^{(\lambda)}\right)=\left(\frac{1-\lambda}{\lambda}\right)^{\mathbb{Z}} \cup\{0\} \\
& S\left(\mathscr{R}_{e}^{(\lambda)}\right)=\left(\frac{1-\lambda}{\lambda}\right)^{2 \mathbb{Z}} \cup\{0\} .
\end{aligned}
$$

where $S(\mathscr{R})$ is the Connes invariant given by the intersection of the spectra of all modular operator determined by faithful normal states of $\mathscr{R}$ (see [23]).

Since by [23] $\sigma_{t}$ is outer unless it is the identity, by (3.7) $\tilde{\gamma}$ is outer and by (3.8') and Lemma $3.1 \mathscr{R}_{e}^{(\lambda)}$ is a $I I I(\lambda /(1-\lambda))^{2}$ factor. Note that this conclusion follows also directly from (3.8') and Lemma 3.1, (ii), Eq. (3.1).

The first equations in (3.8), (3.8') are by now classical [23]; it is likely that proposition 3.2 is also known; we gave details to make our examples explicitly analyzed.

3.3. Corollary. Let $K=N_{1} \oplus N_{2}$, with $N_{1}, N_{2}$ infinite dimensional, and let $E$ denote the orthogonal projection of $K$ onto $N_{1}$. The von Neumann algebra $\pi_{\omega_{\lambda E}}\left(\mathfrak{I}(K)_{e}\right)^{\prime \prime}$ generated by the even subalgebra in the quasifree representation determined by $\lambda E$, is isomorphic to the injective factor of type $I I I_{(\lambda /(1-\lambda))^{2}}$ for $0<\lambda<1 / 2$, and to the injective factor of type $I I_{\infty}$ for $\lambda=1 / 2$.

Proof. Immediate from Lemma 2.1 and Proposition 3.2.

We now specialize $K=N_{1} \oplus N_{2}$ as in Lemma 2.3, so that $\mathfrak{A}(K)_{e}=\mathfrak{B}$ is the $C^{*}$ algebra of quasilocal observables for the free massless Majorana field, and $N_{1}$ is generated by an orthonormal set fulfilling (1.2). Let $E$ be the orthogonal projection on $N_{1}$. 
3.4. Proposition. The representations of $\mathfrak{B}=\mathfrak{U}(K)_{e}$ induced by the states $\omega_{\lambda E} \mid \mathfrak{A}(K)_{e}$ are covariant with positive energy.

Proof. Although this follows from the general statement 2.3, we give an explicit proof. Consider the theory $\mathfrak{U}(K \oplus K)$ with the action $\alpha_{x}$ of the translation group induced by the one particle group representation $\alpha \in \mathbb{R}^{4} \rightarrow \mathscr{U}(x) \oplus \mathscr{U}(x)$, where $\mathscr{U}(x)$ is the translation operator for the Majorana particle on $K$.

Define a self-adjoint projection on $K \oplus K$ by

$$
P_{\lambda}=\left\{\begin{array}{cc}
\lambda E & \lambda^{1 / 2}(1-\lambda)^{1 / 2} E \\
\lambda^{1 / 2}(1-\lambda)^{1 / 2} E & (1-\lambda) E
\end{array}\right\} .
$$

The quasifree state on $\mathfrak{U}(K \oplus K)$ defined by $P_{\lambda}$ in Eq. (3.5) is a pure state $([18])$ whose restriction to $\mathfrak{A}(K \oplus 0)=\mathfrak{A}(K)$ is $\omega_{\lambda E}$. Moreover the range of $P_{\lambda}$ is spanned by the orthonormal set

$$
h_{n}=\lambda^{1 / 2} g_{n} \oplus(1-\lambda)^{1 / 2} g_{n},
$$

which fulfills conditions (1.3). Therefore, by [7] $\pi_{\omega_{p_{\lambda}}}$ is covariant with positive energy and so is $\pi_{\omega_{p_{\lambda}}} \mid \mathfrak{U}(K \oplus 0)$.

Then also $\pi_{\omega_{p_{\lambda}}} \mid \mathfrak{A}(K \oplus 0)_{e}$ is covariant and obeys the spectrum condition. By indentifying $\mathfrak{B}$ with $\mathfrak{U}(K \oplus 0)_{e}$ it is easily seen that $\pi_{\omega p_{\lambda}} \mid \mathfrak{B}$ and the GNS representation of $\omega_{\lambda E} \mid \mathfrak{B}$ are quasiequivalent. Since they both have infinite multiplicity, they are also unitarily equivalent and the assertion follows.

One could similarly construct explicitly type $I I I_{1}$ representations of $\mathfrak{B}$ obeying the spectrum condition, by considering quasifree states $\omega_{A}$, with $A=\lambda_{1} I \oplus \lambda_{2} I \oplus 0$ acting on $K=N_{1} \oplus N_{2} \oplus N_{3}$, where $N_{1}$ and $N_{2}$ are generated each by an infinite orthonormal set fulfilling the conditions 1.3 , and $\lambda_{1} \lambda_{2} \in(0,1 / 2)$ are chosen so that $\left(\lambda_{1} /\left(1-\lambda_{1}\right)\right)^{2}\left(\left(1-\lambda_{2}\right) / \lambda_{2}\right)^{2}$ is not rational.

Acknowledgements. The first named author is grateful to Professor Rudolf Haag for the warm hospitality at Hamburg University, where this work was completed. We are also grateful to Professor Detlev Buchholz for valuable discussions.

\section{References}

1. Haag, R., Kastler, D.: An algebraic approach to Quantum Field Theory, J. Math. Phys. 5, 848 (1964)

2. Araki, H.: On the algebra of all local Observables, Progr. Theor. Phys. 32, 844 (1964)

3. Buchholz, D., Fredenhagen, K.: Locality and the structure of particle states, Commun. Math. Phys. (to appear)

4. Doplicher, S., Powers, R. T.: On the simplicity of the even CAR algebra and free field models, Commun. Math. Phys. 7, 77 (1968)

5. Doplicher, S., Kastler, D., Robinson, D. W.: Covariance algebras in Field Theory and Statistical Mechanics, Commun. Math. Phys. 3, 1 (1966)

6. St $\phi$ rmer, E.: The even CAR algebra, Commun. Math. Phys. 16, 136 (1970)

7. Doplicher, S.: Fock representation and massless particles, Commun. Math. Phys. 3, 328 (1966)

8. Kraus, K., Polley, L., Reents, G.: Generators of infinite direct products of unitary groups, J. Math. Phys. 18, 2166 (1977)

9. Kraus, K., Streater, R. F.: Some covariant representations of massless Fermi Fields preprint

10. Fröhlich, J.: unpublished 
11. Fröhlich, J., Morchio, G., Strocchi, F.: Charged sectors and scattering states in Quantum Electrodynamics, Ann. Phys. 119, 241 (1979)

12. Buchholz, D.: In: Proceedings of the International Conference on Mathematical Physics, Rome 1977, Springer Lecture Notes in Physics Vol 80, Berlin, Heidelberg, New York: Springer 1978

13. Marechal, O.: Une remarque sur un théoreme de Glimm, Bull. Sci. Math. 99, 41 (1975)

14. Glimm, J.: A Stone Weierstrass theorem for $C^{*}$ algebras, Ann. Math. 72, 216 (1960)

15. Dixmier, J.: $C^{*}$ algebras, Amsterdam: North Holland 1980

16. Borchers, H. J.: In Cargese Lectures on Theoretical Physics 1969, D. Kastler, (ed.) New York, London, Paris: Gordon and Breach, 1970

17. Borchers, H. J.: Energy and momentum as observables, Commun. Math. Phys. 2, 49 (1966)

18. Powers, R., St $\phi$ rmer, E.: Free states of the canonical anti-commutation relations, Commun. Math. Phys. 16, 1 (1970)

19. Connes, A.: In: $C^{*}$ algebre e le loro applicazioni in Fisica Teorica; and Teoria $\mathrm{K}$, algebre di operatori e teoria dell'indice, Symposia Mathematica Vol. 20, London and New York: Academic Press 1976

20. Takesaki, M.: Conditional expectation in von Neumann algebras, J. Funct. Anal. 9, 306 (1972)

21. Araki, H.: Remarks on Spectra of modular operators of von Neumann algebras, Commun. Math. Phys. 28, 267 (1972)

22. Takesaki, M.: Tomita's theory of modular Hilbert algebras, In: Lecture Notes in Mathematics Vol. 128, Berlin, Heidelberg, New York: Springer 1970

23. Connes, A.: In: $C^{*}$ algebras and their applications in Field Theory and Statistical Mechanics, Varenna School 1973, Amsterdam: North Holland 1976

24. Rocca, F., Sirugue, M., Testard, D.: On a Class of Equilibrium States under the Kubo-MartinSchwinger condition. I. Fermions, Commun. Math. Phys. 13, 317 (1969)

Communicated by R. Haag

Received November 10, 1981 
